\title{
Monomeric Ti(IV) homopiperazine complexes and their exploitation for the ring opening polymerisation of rac-lactide
}

\author{
Stuart L Hancock, Mary F Mahon and Matthew D Jones ${ }^{*}$
}

\begin{abstract}
Background: The area of biodegradable/sustainable polymers is one of increasing importance in the 21st Century due to their positive environmental characteristics. Lewis acidic metal centres are currently one of the most popular choices for the initiator for the polymerisation. Thus, in this paper we report the synthesis and characterisation of a series of monometallic homopiperazine Ti(IV) complexes where we have systematically varied the sterics of the phenol moieties.

Results: When the ortho substituent of the ligand is either a Me, tBu or amyl then the $\beta$-cis isomer is isolated exclusively in the solid-state. Nevertheless, in solution multiple isomers are clearly observed from analysis of the NMR spectra. However, when the ortho substituent is an H-atom then the trans-isomer is formed in the solid-state and solely in solution. The complexes have been screened for the polymerisation of rac-lactide in solution and under the industrially preferred melt conditions. Narrow molecular weight material (PDI $1.07-1.23$ ) is formed under melt conditions with controlled molecular weights.

Conclusions: Six new Ti(IV) complexes are presented which are highly active for the polymerisation. In all cases atactic polymer is prepared with predictable molecular weight control. This shows the potential applicability of Ti(IV) to initiate the polymerisations.
\end{abstract}

Keywords: Titanium, Polymerisation, Lactide, Catalysis

\section{Background}

As part of our on-going studies into the chemistry of group 4 metals and homo/piperazine derived salan ligands [1-3] in this paper we report the synthesis and characterisation of series of monometallic complexes based on the homopiperazine backbone. This ligand family has also been applied to $\mathrm{Fe}(\mathrm{III})$ [4,5], $\mathrm{Cu}$ (II) [6], $\mathrm{Ni}(\mathrm{II})$ [6] and $\mathrm{Mo}(\mathrm{VI})$ [7] metal centres. Typically these are either monomeric or dimeric structures in the solid-state. These 7-membered ring ligands are underrepresented in the literature compared to their 6membered brothers or their linear amine bis(phenolate) cousins [8-13]. To re-address this imbalance we have previously reported the formation of $\mathrm{Ti}_{2}\left(\mathrm{O}^{\mathrm{i}} \mathrm{Pr}\right)_{6} \mathrm{~L}$ or monometallic $\mathrm{Zr} / \mathrm{Hf}\left(\mathrm{O}^{\mathrm{i}} \mathrm{Pr}\right)_{2} \mathrm{~L}$ species (containing homopiperazine salan ligands) where, in the monometallic

\footnotetext{
* Correspondence: mj205@bath.ac.uk

Department of Chemistry, University of Bath, Claverton Down, Bath BA2 7AY, UK
}

examples, the OiPr moieties are trans to one another [3]. Utilising the piperazine derived salan ligands with $\mathrm{Zr}$ (IV) and $\mathrm{Hf}(\mathrm{IV})$ starting materials leads to unpredictable reactions with no rationale control over the product formed [3]. These complexes have been shown to be effective initiators for the ring opening polymerisation (ROP) of cyclic esters [2,3]. Moreover, we have prepared Al(III) complexes of homopiperazine salan ligands for co-polymerisations of cyclic esters [1]. The rich and unexplored chemistry of this ligand set motivated us to prepare monometallic Ti(IV) complexes for the controlled ROP of rac-lactide. The driving force for this work also lies in the attractive properties of the final polymer polylactide (PLA) itself, such as biodegradability, it is produced from annually renewable resources and the fact that the polymer is also biocompatible [14]. These facets have spear-headed research in this area and metals such as $\mathrm{Ca}$ (II) [15,16], $\mathrm{Mg}$ (II) [17-20], $\mathrm{Zn}$ (II) [21-27], $\mathrm{Al}(\mathrm{III})$ [28-36], Bi(III) [37], Ti(IV)/Zr(IV) [38-40] and metal-free systems [41-43] have all proved excellent 
choices in the literature. The controlled polymerisation of rac-lactide can lead to either atactic, heterotactic or isotactic PLA the later possessing a significantly higher melting temperature. There is an exigent desire to prepare and characterise new initiators for the ROP of lactide to enhance the already impressive properties of the material. A selection of complexes for the polymerisation of rac-lactide is shown in Figure 1. One of the earliest examples of the ROP of rac-lactide was by Spassky and co-workers [33], they produced isotactically enriched PLA with an Aluminium Schiff base complex. Then followed seminal studies on Zn-BDI complexes [17], in solution with a monomer: initiator ratio of $200: 1$ at $20^{\circ} \mathrm{C}$ heterotactic PLA $P_{r}=0.90$ was produced. There is a desire to move towards melt polymerisations, in the absence of solvent. One of the first examples of this approach was the work of Feijen [35], who produced highly isotactically enriched PLA from raclactide at $130^{\circ} \mathrm{C}$ (monomer:initiator 200:1), however to achieve high conversions 48 hours was required. Davidson has shown that it is possible to produce heterotactically $\left(P_{r}=0.90\right)$ enriched PLA in the melt with a group 4 amine tris(phenolate) complex (monomer:initiator 300:1), near quantitative conversion was achieved after 10 minutes [44].

\section{Results and discussion}

\section{Complex preparation}

Literature preparation methods were utilised to prepare the homopiperazine salan ligands, $(\mathbf{1 - 6}) \mathrm{H}_{2}[3,45]$. The complexes were prepared by a $1: 1$ reaction of the salan with $\mathrm{Ti}\left(\mathrm{O}^{\mathrm{i}} \mathrm{Pr}\right)_{4}$ at $80^{\circ} \mathrm{C}$, this was carried out under a flow of Ar to facilitate the removal of isopropanol to drive the reaction to the formation of the 1:1 complex. The additional heating $\left(80^{\circ} \mathrm{C}\right)$ allowed the homopiperazine ring backbone to adopt the thermodynamically unfavourable boat type configuration and furthermore coordinate both phenols and nitrogen centres to a single titanium metal centre, Scheme 1. These complexes were characterised by elemental analysis, ${ }^{1} \mathrm{H},{ }^{13} \mathrm{C}\left\{{ }^{1} \mathrm{H}\right\}$ NMR spectroscopy and where possible single crystal X-ray diffraction.

The solid-state structures $\mathrm{Ti}(\mathbf{2 , 4 - 6})\left(\mathrm{O}^{\mathrm{i}} \mathrm{Pr}\right)_{2}$ have been determined by single crystal X-ray diffraction, and have yielded monometallic complexes with the titanium metal centres adopting a pseudo octahedral configuration. The structure obtained for $\mathrm{Ti}(2)\left(\mathrm{O}^{\mathrm{i}} \mathrm{Pr}\right)_{2}$ is given as a representative example (Figure 2). $\mathrm{Ti}(\mathbf{2}, \mathbf{4}, \mathbf{5})\left(\mathrm{O}^{\mathrm{i}} \mathrm{Pr}\right)_{2}$ adopt a $\beta$-cis configuration in the solid-state, this is in contrast to the $\mathrm{Zr}(\mathrm{IV}) / \mathrm{Hf}(\mathrm{IV})$ analogues which formed trans complexes. However, with less steric bulk in the ortho-phenol position a pseudo trans-octahedral titanium complex supported by a homopiperazine salan ligand, $\mathrm{Ti}(6)\left(\mathrm{O}^{\mathrm{i}} \mathrm{Pr}\right)_{2}$ (Figure 3 ), was isolated.

Selected bond lengths $(\AA)$ and angles $\left(^{\circ}\right)$ are given in Table 1 for the crystallographically characterised titanium homopiperazine complexes. Those complexes which adopted a $\beta$-cis configuration $\left\{\mathrm{Ti}(\mathbf{2 , 4 - 6})\left(\mathrm{O}^{\mathrm{i}} \mathrm{Pr}\right)_{2}\right\}$ revealed similar bond lengths and angles. There was no significant difference in the isopropoxide metal (Ti1-O1, Ti1O2) bond lengths, but phenoxy-metal bond lengths (Ti1-O3, Ti1-O4) were significantly different with the phenoxy trans to an isopropoxide exhibiting a longer bond length. The two Ti-N bonds are different with Ti$\mathrm{N}$ trans to an isopropoxide being the longer distance.

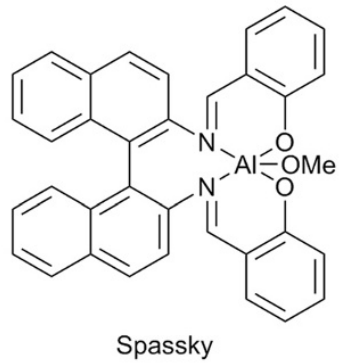

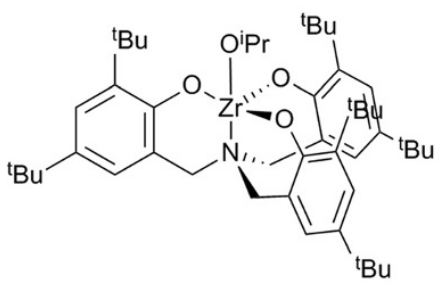

Davidson
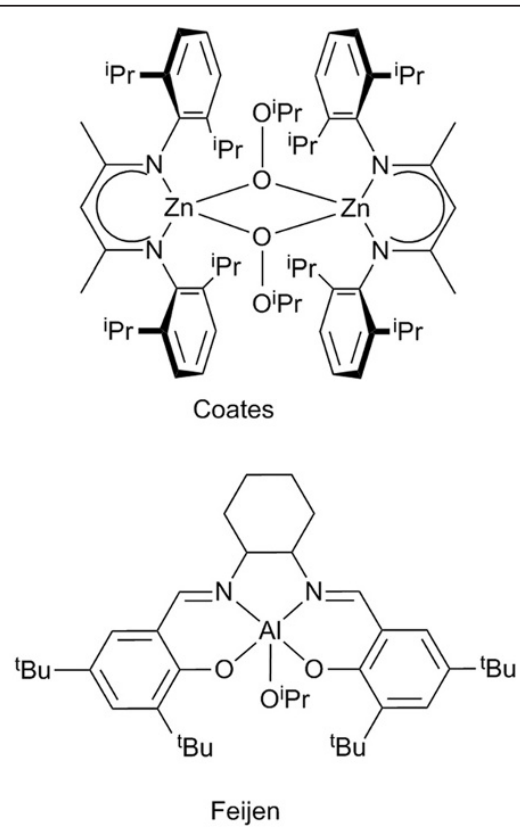

Figure 1 Examples of initiators for the ROP of rac-lactide. 
<smiles>[R]c1cc([R])c(O)c(CN2CCCN(Cc3cc([R])cc(Br)c3O)CC2)c1</smiles>

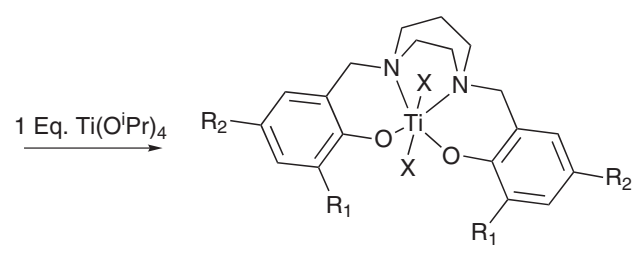

$$
\begin{array}{ll} 
& \mathrm{R}_{1} \quad \mathrm{R}_{2} \\
\mathrm{Ti}(1)\left(\mathrm{O}^{\mathrm{i} P r}\right)_{2} & \mathrm{Me}, \mathrm{Me} \\
\mathrm{Ti}(2)\left(\mathrm{O}^{\mathrm{i} P r}\right)_{2} & \mathrm{Me},{ }^{\mathrm{Bu}} \\
\mathrm{Ti}(3)\left(\mathrm{O}^{\mathrm{i} P}\right)_{2} & { }^{\mathrm{t}} \mathrm{Bu},{ }^{\mathrm{B} u} \\
\mathrm{Ti}(4)\left(\mathrm{O}^{\mathrm{i} P r}\right)_{2} & { }^{\mathrm{t}} \mathrm{Bu}, \mathrm{Me} \\
\mathrm{Ti}(5)\left(\mathrm{O}^{\mathrm{i} P r}\right)_{2} & \mathrm{Amyl}, \mathrm{Amyl} \\
\mathrm{Ti}(6)\left(\mathrm{O}^{\mathrm{i}} \mathrm{Pr}\right)_{2} & \mathrm{H}, \quad{ }^{\mathrm{t}} \mathrm{Bu} \\
\text { where X }=\mathrm{O}^{\mathrm{i} P r} &
\end{array}
$$

Scheme 1 Synthesis of titanium monometallic complexes supported by homopiperazine salan ligands.

The complexes adopted a distorted octahedral conformation, which is demonstrated by the deviation of the titanium angles from $90^{\circ}$ or $180^{\circ}$, for cis or trans angles respectively. A high degree of variation from the idealistic $90^{\circ}$ angle was observed between N1-Ti1-N2, giving angles between $67.90(13)-68.08(7)^{\circ}$.

The less sterically hindered salan complex with hydrogen atoms at the ortho positions adopted a distorted trans-octahedral structural configuration $\left\{\mathrm{Ti}(\mathbf{6})\left(\mathrm{O}^{\mathrm{i}} \mathrm{Pr}\right)_{2}\right\}$ (Figure 3). The two phenoxy-titanium bonds (Ti1-O1, Ti1-O2) are equivalent in length, additionally the two nitrogen-titanium bonds (Ti1-N1, Ti1-N2) are equivalent in length. This is indicative of the structures symmetrical nature. Similar to $\beta$-cis configurations the trans-octahedral structure deviates from an ideal octahedral environment.

The solution-state NMR spectra for the monometallic titanium piperazine salan complexes $\mathrm{Ti}(\mathbf{1}-\mathbf{5})\left(\mathrm{O}^{\mathrm{i}} \mathrm{Pr}\right)_{2}$ show that the complexes adopt multiple conformations in solution, unlike the solid-state structures which all showed the $\beta$-cis conformation. For example for $\operatorname{Ti}(\mathbf{1}-\mathbf{2})$ $\left(\mathrm{O}^{\mathrm{i}} \mathrm{Pr}\right)_{2}$ two conformations are observed in solution. One of the two species in solution is comparatively well defined whereas the other is fluxional. For example

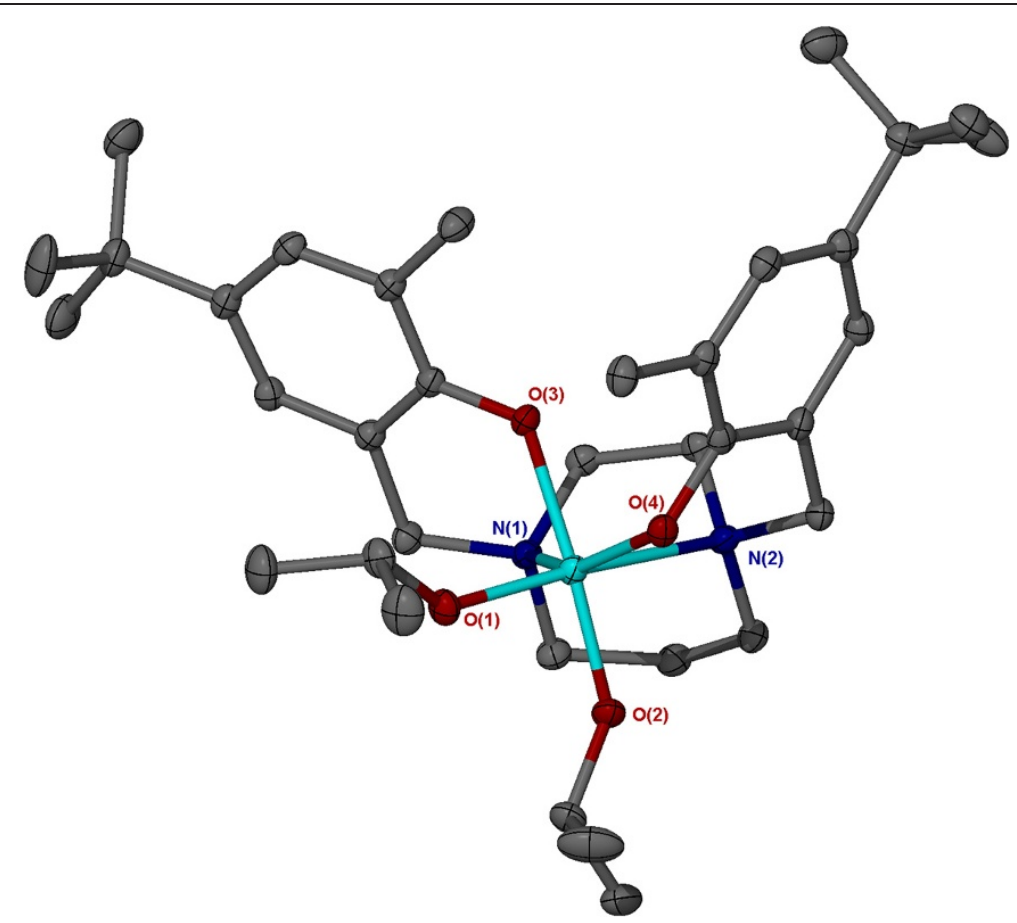

Figure 2 Solid-state structure for $\mathbf{T i}(2)\left(\mathbf{O}^{\mathrm{i}} \mathrm{Pr}\right)_{2}$ in the $\boldsymbol{\beta}$-cis configuration. Ellipsoids are shown at the $30 \%$ probability level, hydrogen atoms have been removed for clarity. 


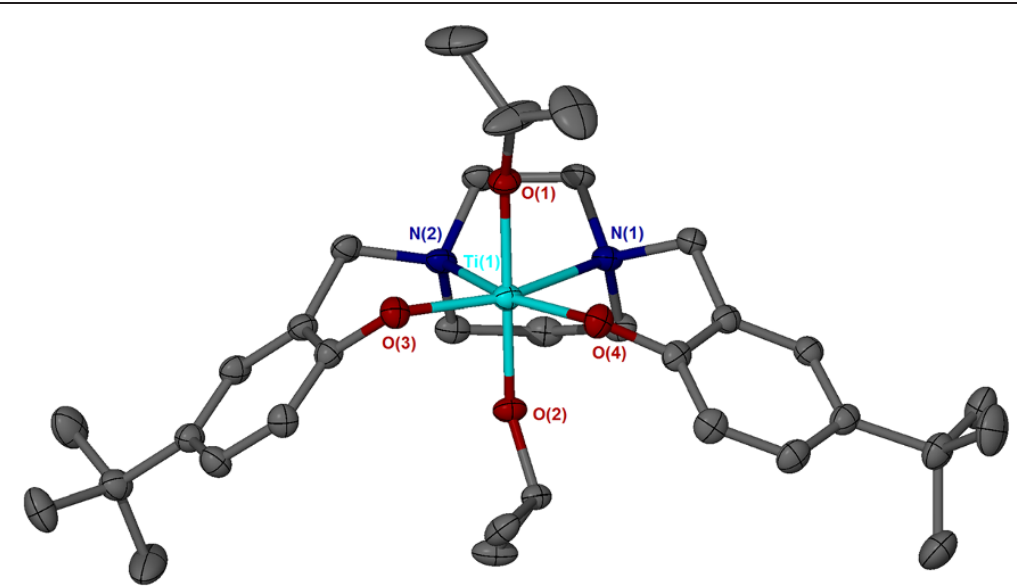

Figure 3 Solid-state structure for $\mathrm{Ti}(6)\left(\mathbf{O}^{i} \mathrm{Pr}\right)_{2}$ in the trans-configuration. Ellipsoids are shown at the $30 \%$ probability level, hydrogen atoms have been removed for clarity.

isopropoxide $-\mathrm{CH}_{3}$ resonances were located at $0.40 \mathrm{ppm}$ and $1.19 \mathrm{ppm}$ (presumably the $\beta$-cis isomer) and a broad resonance was further observed between $0.45-1.55 \mathrm{ppm}$. The fluxional nature is supported by variable temperature NMR spectroscopy (233 K) where the resonances become much more defined at lower temperatures. These complexes can adopt the $\alpha$-cis, $\beta$-cis, and trans octahedral conformations. Although the $\Delta$ and $\Lambda$ forms of $\alpha$-cis and $\beta$-cis conformations are possible they are indistinguishable by conventional NMR spectroscopy (Figure 4) [46]. It should be noted that although three octahedral conformations are present the orientation of the homopiperazine ring can further complicate the NMR spectra.

The more sterically hindered complexes $\operatorname{Ti}(\mathbf{3}-\mathbf{5})$ $\left(\mathrm{O}^{\mathrm{i}} \mathrm{Pr}\right)_{2}$, with respect to the ortho-phenoxy positions, primarily adopted two conformations. The two conformations can be observed in their NMR spectra. For example for $\mathrm{Ti}(3)(\mathrm{O} \mathrm{Pr})_{2}$ the isopropoxide $-\mathrm{CH}_{3}$ region

Table 1 Selected bond lengths $(\AA)$ and angles $\left(^{\circ}\right)$ for $\mathrm{Ti}$ $(2,4-6)\left(O^{i} \mathrm{Pr}\right)_{2}$, as determined by $\mathrm{X}$-ray diffraction studies

\begin{tabular}{|c|c|c|c|c|}
\hline & $\mathrm{Ti}(2)\left(\mathrm{O}^{\mathrm{i}} \mathrm{Pr}\right)_{2}$ & $\mathrm{Ti}(4)\left(\mathrm{O}^{\mathrm{i}} \mathrm{Pr}\right)_{2}$ & $\mathrm{Ti}(5)\left(\mathrm{O}^{\mathrm{i}} \mathrm{Pr}\right)_{2}$ & $\mathrm{Ti}(6)\left(\mathrm{O}^{\mathrm{i}} \mathrm{Pr}\right)_{2}$ \\
\hline Ti1-01 & $1.8310(17)$ & $1.836(4)$ & $1.812(3)$ & $1.8490(18)$ \\
\hline $\mathrm{Ti1}-\mathrm{O} 2$ & $1.8375(16)$ & $1.833(3)$ & $1.838(3)$ & $1.8323(17)$ \\
\hline Ti1-03 & $1.9568(16)$ & $1.931(3)$ & $1.939(3)$ & 1.9175(19) \\
\hline Ti1-O4 & $1.8834(17)$ & $1.873(3)$ & $1.892(3)$ & $1.9106(18)$ \\
\hline Ti1-N1 & $2.285(2)$ & $2.293(4)$ & $2.298(4)$ & $2.255(2)$ \\
\hline Ti1-N2 & $2.349(2)$ & $2.346(4)$ & $2.334(4)$ & $2.268(2)$ \\
\hline N1-Ti1-O1 & 101.94(7) & 102.78(16) & 103.54(15) & $86.18(8)$ \\
\hline N1-Ti1-O2 & $92.39(7)$ & $89.46(16)$ & $89.09(14)$ & $90.87(8)$ \\
\hline N2-Ti1-O1 & 168.57(8) & $170.35(16)$ & $171.26(14)$ & $88.51(8)$ \\
\hline N2-Ti1-O2 & $84.54(7)$ & $86.07(15)$ & $86.41(14)$ & $87.99(8)$ \\
\hline N1-Ti1-N2 & $68.08(7)$ & $67.91(15)$ & $67.90(13)$ & 70.09(8) \\
\hline
\end{tabular}

shows doublets at $0.39 \mathrm{ppm}, 0.98 \mathrm{ppm}$ and $1.01 \mathrm{ppm}$ which are related to one conformation. The analogous resonances are present from the other conformation at $0.55 \mathrm{ppm}, 0.72 \mathrm{ppm}, 0.94 \mathrm{ppm}$, and $0.97 \mathrm{ppm}$ (each a $3 \mathrm{H}$ integral) respectively. The two species were present in an approximate 1:0.9 ratio. The same can be observed in the aromatic region where resonances at $6.88 \mathrm{ppm}$, and $7.25 \mathrm{ppm}$ were attributed to the slightly dominant conformation. The ${ }^{1} \mathrm{H}$ NMR resonances are relatively well defined for each conformation at room temperature, it was speculated that the increased steric demands of the ligands reduce fluxionality within the complex when compared to $\mathrm{Ti}(\mathbf{1}-\mathbf{2})\left(\mathrm{O}^{\mathrm{i}} \mathrm{Pr}\right)_{2}$.

The less sterically hindered $\operatorname{Ti}(\mathbf{6})\left(\mathrm{O}^{\mathrm{i}} \mathrm{Pr}\right)_{2}$ exclusively formed the trans octahedral conformation in solution and the solid-state, as determined by ${ }^{1} \mathrm{H} /{ }^{13} \mathrm{C}\left\{{ }^{1} \mathrm{H}\right\}$ NMR spectroscopy and single crystal $\mathrm{X}$-ray diffraction. The isopropoxide $-\mathrm{CH}_{3}$ protons afforded only two resonances at $0.63 \mathrm{ppm}$ and $1.15 \mathrm{ppm}$ (both $6 \mathrm{H}$ integrals)

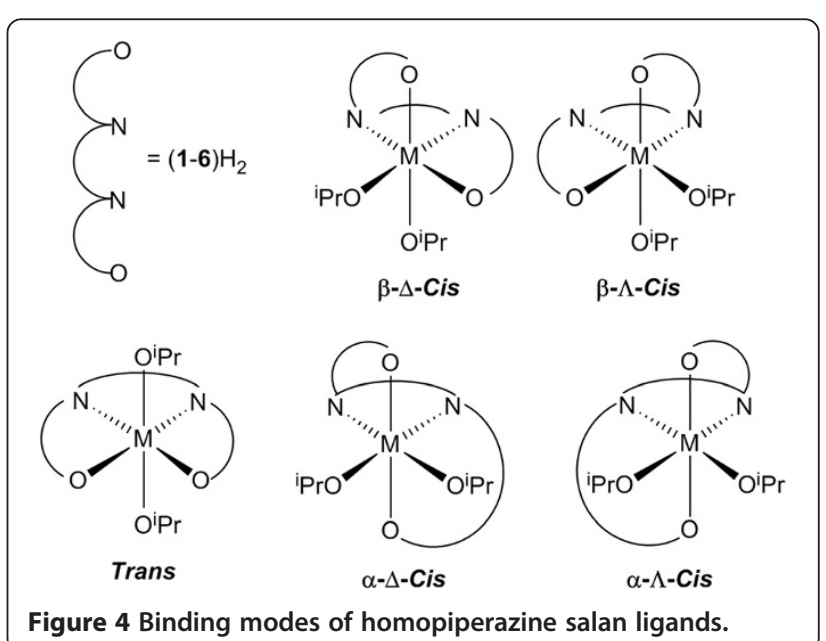


thus consistent with a trans octahedral geometry being formed exclusively. This is further supported by the presence of two isopropoxide septets at $3.84 \mathrm{ppm}$ and $4.82 \mathrm{ppm}$.

\section{Polymerisation studies}

The isolated $\operatorname{Ti}(\mathbf{1}-\mathbf{6})\left(\mathrm{O}^{\mathrm{i}} \mathrm{Pr}\right)_{2}$ complexes were trialled for the ROP of rac-lactide in toluene $(10 \mathrm{ml})$ at $80^{\circ} \mathrm{C}$ at a 100:1 [rac-lactide]:[Initiator] ratio (Table 2). Limited activity was observed for this initiator series under these conditions typically achieving low conversions after $24 \mathrm{~h}$. The molecular weights were consistent with one PLA chain per metal; additionally PDI values were low indicating a more controlled polymerisation system than their bimetallic counterparts [2]. The monometallic system is stable at $80^{\circ} \mathrm{C}$ therefore it was assumed the monometallic species were initiating the polymerisation reaction. Where the initiators were active enough to obtain reliable $P_{r}$ values a slight isotactic bias was observed.

$\mathrm{Ti}(\mathbf{1}-\mathbf{6})\left(\mathrm{O}^{\mathrm{i}} \mathrm{Pr}\right)_{2}$ titanium salan complexes were trialled for the ROP of $\mathrm{rac}$-lactide without solvent at $130^{\circ} \mathrm{C}$ at a 300:1 [rac-lactide]:[Initiator] ratio (Table 3). Under solvent free conditions these initiators typically achieved 41-60\% conversion after $24 \mathrm{~h}$. Despite the presence of two potentially initiating isopropoxide groups per metal the PDI values remained low $(\mathrm{PDI}<1.25)$ at the elevated temperature. The defined structure permits the formation of controlled PLA chains but the lack of flexibility within the molecules causes the initiators to be hindered thus leading to reduced activity. Under melt conditions $\mathrm{Ti}(\mathbf{1}-\mathbf{6})\left(\mathrm{O}^{\mathrm{i}} \mathrm{Pr}\right)_{2}$ complexes produced PLA with a slight heterotactic bias $\left(P_{r}=0.51-0.63\right)$. The steric effects do not seem to significantly alter the polymerisation, with the more bulky amyl substituted complex being more active than the sterically unhindered $\operatorname{Ti}(\mathbf{6})\left(\mathrm{O}^{\mathrm{i}} \mathrm{Pr}\right)_{2}$ complex.

\section{Conclusions}

In conclusion a series of six new $\mathrm{Ti}(\mathrm{IV})$ complexes have been prepared based on a homopiperazine salan derived ligand. In solution a multitude of species are formed.

Table 2 Solution ROP of rac-lactide for $\mathrm{Ti}(1-6)\left(0^{\mathrm{i}} \mathrm{Pr}\right)_{2}$ in $10 \mathrm{ml}$ of toluene at $80^{\circ} \mathrm{C}$ in a $100: 1$ [rac-lactide]:[initiator]

\begin{tabular}{lccccc}
\hline & Time (hours) & Conv. $^{(\%)^{\mathbf{a}}}$ & $\boldsymbol{M}_{\boldsymbol{n}}^{\boldsymbol{b}}$ & $\mathbf{P D I}^{\mathbf{b}}$ & $\boldsymbol{P}_{\boldsymbol{r}}^{\boldsymbol{c}}$ \\
\hline $\mathrm{Ti}(\mathbf{1})\left(\mathrm{O}^{\mathrm{i}} \mathrm{Pr}\right)_{2}$ & 24 & 23 & 1250 & 1.63 & $-_{-}^{\mathrm{d}}$ \\
$\mathrm{Ti}(\mathbf{2})\left(\mathrm{O}^{\mathrm{i}} \mathrm{Pr}\right)_{2}$ & 24 & 8 & - & - & $-{ }^{\mathrm{d}}$ \\
$\mathrm{Ti}(\mathbf{3})\left(\mathrm{O}^{\mathrm{i}} \mathrm{Pr}\right)_{2}$ & 24 & 27 & 4700 & 1.17 & $-_{\mathrm{d}}$ \\
$\mathrm{Ti}(\mathbf{4})\left(\mathrm{O}^{\mathrm{i}} \mathrm{Pr}\right)_{2}$ & 24 & 12 & 700 & 1.01 & $-_{-\mathrm{d}}$ \\
$\mathrm{Ti}(\mathbf{5})\left(\mathrm{O}^{\mathrm{i}} \mathrm{Pr}\right)_{2}$ & 24 & 50 & 8200 & 1.06 & 0.44 \\
$\mathrm{Ti}(\mathbf{6})(\mathrm{O} \mathrm{Pr})_{2}$ & 24 & 32 & 6950 & 1.11 & 0.44 \\
\hline
\end{tabular}

${ }^{\mathrm{a}}$ Conversion ascertained by ${ }^{1} \mathrm{H}$ NMR spectroscopy. ${ }^{\mathrm{b}}$ Molecular weight and PDI determined by GPC (THF) using polystyrene standards. ${ }^{C} P_{r}$ as calculated from ${ }^{1} \mathrm{H}$ NMR homonuclear decoupled spectroscopy in $\mathrm{CDCl}_{3} \cdot{ }^{\mathrm{d}} P_{r}$ could not be accurately determined, strong tacticity was not observed.
Table 3 Solvent free ROP of rac-lactide for $\mathrm{Ti}(1-6)\left(\mathrm{O}^{\mathrm{P}} \mathrm{Pr}\right)_{2}$ at $130^{\circ} \mathrm{C}$ in a $300: 1$ [rac-lactide]:[initiator]

\begin{tabular}{lccccc}
\hline & Time (hours) & Conv. (\%) & $\boldsymbol{M}_{\boldsymbol{n}}^{\boldsymbol{b}}$ & PDI $^{\mathbf{b}}$ & $\boldsymbol{P}_{\boldsymbol{r}}^{\boldsymbol{c}}$ \\
\hline $\mathrm{Ti}(\mathbf{1})\left(\mathrm{O}^{\mathrm{i}} \mathrm{Pr}\right)_{2}$ & 24 & 54 & 12050 & 1.07 & 0.56 \\
$\mathrm{Ti}(\mathbf{2})\left(\mathrm{O}^{\mathrm{P}} \mathrm{Pr}\right)_{2}$ & 24 & 50 & 7900 & 1.19 & 0.63 \\
$\mathrm{Ti}(\mathbf{3})\left(\mathrm{O}^{\mathrm{i}} \mathrm{Pr}\right)_{2}$ & 24 & 42 & 7050 & 1.14 & 0.53 \\
$\mathrm{Ti}(\mathbf{4})\left(\mathrm{O}^{\mathrm{P}} \mathrm{Pr}\right)_{2}$ & 24 & 41 & 6750 & 1.10 & 0.63 \\
$\mathrm{Ti}(\mathbf{5})\left(\mathrm{O}^{\mathrm{i}} \mathrm{Pr}\right)_{2}$ & 24 & 60 & 10900 & 1.14 & 0.61 \\
$\mathrm{Ti}(\mathbf{6})\left(\mathrm{O}^{\mathrm{i}} \mathrm{Pr}\right)_{2}$ & 24 & 51 & 6850 & 1.23 & 0.55 \\
\hline
\end{tabular}

${ }^{a}$ Conversion ascertained by ${ }^{1} \mathrm{H}$ NMR spectroscopy. ${ }^{\mathrm{b}}$ Molecular weight and PDI determined by GPC (THF) using polystyrene standards. ${ }^{C} P_{r}$ as calculated from ${ }^{1} \mathrm{H}$ NMR homonuclear decoupled spectroscopy in $\mathrm{CDCl}_{3}$.

However, in the solid-state the $\beta$-cis and trans forms were observed, depending on the steric requirement of the ligand. All complexes were active for the ROP of rac-LA in solution and under the industrially preferred melt conditions.

\section{Experimental}

$\mathrm{Ti}(\mathbf{1})\left(\mathrm{O}^{\mathrm{i}} \mathrm{Pr}\right)_{2} \cdot \mathbf{1 H}_{2}(0.37 \mathrm{~g}, 1.00 \mathrm{mmol})$ and $\mathrm{Ti}\left(\mathrm{O}^{\mathrm{i}} \mathrm{Pr}\right)_{4}$ $(0.30 \mathrm{ml}, 1.01 \mathrm{mmol})$ were dissolved in toluene $(30 \mathrm{ml})$ then heated $\left(80^{\circ} \mathrm{C}\right)$ and stirred $(16 \mathrm{~h})$. The solvent was removed in-vacuo and recrystallised from hexane to yield pale yellow crystals ( $0.14 \mathrm{~g}, 0.26 \mathrm{mmol}, 26 \%) .2$ species identified in the solution state NMR spectra. ${ }^{1} \mathrm{H}$ $\operatorname{NMR}\left(\mathrm{CDCl}_{3}\right): \delta 0.40\left(3 \mathrm{H}, \mathrm{d}, \mathrm{J}=5.5 \mathrm{~Hz}, \mathrm{CH}_{3}\right), 1.14(6 \mathrm{H}$, br, $\left.\mathrm{CH}_{3}\right), 1.19\left(3 \mathrm{H}, \mathrm{d}, \mathrm{J}=5.5 \mathrm{~Hz}, \mathrm{CH}_{3}\right), 1.68(1 \mathrm{H}, \mathrm{m}$, $\left.\mathrm{CH}_{2}\right), 1.88\left(1 \mathrm{H}, \mathrm{m}, \mathrm{CH}_{2}\right), 2.21\left(9 \mathrm{H}, \mathrm{s}, \mathrm{CH}_{3}\right), 2.29(3 \mathrm{H}, \mathrm{s}$, $\left.\mathrm{CH}_{3}\right), 2.42\left(2 \mathrm{H}, \mathrm{br}, \mathrm{CH}_{2}\right), 2.79\left(1 \mathrm{H}, \mathrm{d}, \mathrm{J}=6.0 \mathrm{~Hz}, \mathrm{CH}_{2}\right)$, $3.11\left(1 \mathrm{H}, \mathrm{d}, \mathrm{J}=11.5 \mathrm{~Hz}, \mathrm{CH}_{2}\right), 3.31\left(2 \mathrm{H}, \mathrm{s}, \mathrm{CH}_{2}\right), 3.60$ $\left(1 \mathrm{H}, \mathrm{d}, \mathrm{J}=6.5 \mathrm{~Hz}, \mathrm{CH}_{2}\right), 3.72\left(1 \mathrm{H}, \mathrm{m}, \mathrm{CH}_{2}\right), 3.95(1 \mathrm{H}, \mathrm{m}$, $\left.\mathrm{CH}_{2}\right), 4.20\left(2 \mathrm{H}, \mathrm{d}, \mathrm{J}=11.0 \mathrm{~Hz}, \mathrm{CH}_{2}\right), 4.46\left(1 \mathrm{H}, \mathrm{m}, \mathrm{CH}_{2}\right)$, $4.85\left(1 \mathrm{H}, \mathrm{m}, \mathrm{CH}_{2}\right), 4.93\left(1 \mathrm{H}, \mathrm{m}, \mathrm{CH}_{2}\right), 6.68(2 \mathrm{H}, \mathrm{s}, \mathrm{ArH})$, $6.91(1 \mathrm{H}, \mathrm{s}, \mathrm{ArH}) .2$ nd species ${ }^{1} \mathrm{H}$ NMR $\left(\mathrm{CDCl}_{3}\right): \delta 0.45-$ $1.45\left(12 \mathrm{H}, \mathrm{br}, \mathrm{CH}_{3}\right), 2.00-2.50\left(12 \mathrm{H}, \mathrm{br}, \mathrm{CH}_{3}\right), 2.00-$ 2.50 (4H, br, $\left.\mathrm{CH}_{2}\right), 3.00-5.00\left(10 \mathrm{H}, \mathrm{br}, \mathrm{CH}_{2}\right), 3.00-5.00$ (2H, br, CH), 6.58 (2H, s, ArH ), 6.87 (2H, s, ArH). ${ }^{13} \mathrm{C}$ $\left\{{ }^{1} \mathrm{H}\right\}$ NMR $\left(\mathrm{CDCl}_{3}\right): \delta 16.5\left(\mathrm{CH}_{3}\right), 16.9\left(\mathrm{CH}_{3}\right), 20.8\left(\mathrm{CH}_{3}\right)$, $23.0\left(\mathrm{CH}_{2}\right), 23.7\left(\mathrm{CH}_{2}\right), 25.9\left(\mathrm{CH}_{3}\right), 26.1\left(\mathrm{CH}_{3}\right), 26.3$ $\left(\mathrm{CH}_{3}\right), 50.8\left(\mathrm{br}, \mathrm{CH}_{2}\right), 55.6\left(\mathrm{CH}_{2}\right), 58.0\left(\mathrm{CH}_{2}\right), 59.2(\mathrm{br}$, $\left.\mathrm{CH}_{2}\right), 62.7$ (br, $\left.\mathrm{CH}_{2}\right), 64.1\left(\mathrm{CH}_{2}\right), 72.1(\mathrm{CH}), 73.2(\mathrm{CH})$, $75.7(\mathrm{CH}), 75.9(\mathrm{CH}), 123.4(\mathrm{ArH}), 124.6(\mathrm{ArH}), 125.4$ (ArH), 127.4 (Ar), 122.0 - 132.0 (Ar), 131.5 (Ar), 163.0 (ArO). Calc. (\%) for $\mathrm{C}_{29} \mathrm{H}_{44} \mathrm{~N}_{2} \mathrm{O}_{4} \mathrm{Ti}$ : C 65.41, $\mathrm{H}$ 8.33, N 5.26. Found (\%), C 65.29, H 8.27, N 5.37.

$\mathrm{Ti}(2)\left(\mathrm{O}^{\mathrm{i}} \mathrm{Pr}\right)_{2} .2 \mathrm{H}_{2}(0.46 \mathrm{~g}, 1.02 \mathrm{mmol})$ and $\mathrm{Ti}\left(\mathrm{O}^{\mathrm{i}} \mathrm{Pr}\right)_{4}$ $(0.30 \mathrm{ml}, 1.01 \mathrm{mmol})$ were dissolved in toluene $(30 \mathrm{ml})$ then heated $\left(80^{\circ} \mathrm{C}\right)$ and stirred $(16 \mathrm{~h})$. The solvent was removed in-vacuo and recrystallised from hexane to yield pale yellow crystals $(0.48 \mathrm{~g}, 0.78 \mathrm{mmol}, 77 \%)$. 2 species identified in the solution state NMR spectra. ${ }^{1} \mathrm{H}$ NMR $\left(\mathrm{CDCl}_{3}\right): \delta 0.32\left(3 \mathrm{H}, \mathrm{d}, \mathrm{J}=6.0 \mathrm{~Hz}, \mathrm{CH}_{3}\right), 0.87(6 \mathrm{H}, \mathrm{br}$, $\left.\mathrm{CH}_{3}\right), 1.65\left(9 \mathrm{H}, \mathrm{d}, \mathrm{J}=6.0 \mathrm{~Hz}, \mathrm{CH}_{3}\right), 1.26\left(36 \mathrm{H}, \mathrm{s}, \mathrm{CH}_{3}\right)$, 
$1.71\left(1 \mathrm{H}, \mathrm{m}, \mathrm{CH}_{2}\right), 1.90\left(2 \mathrm{H}, \mathrm{m}, \mathrm{CH}_{2}\right), 2.16$ (4H, br, $\mathrm{CH}_{2}$ ), $2.28\left(12 \mathrm{H}, \mathrm{s}, \mathrm{CH}_{3}\right), 2.24\left(2 \mathrm{H}, \mathrm{br}, \mathrm{CH}_{2}\right), 2.80(1 \mathrm{H}, \mathrm{d}, \mathrm{J}=6.5$ $\left.\mathrm{Hz}, \mathrm{CH}_{2}\right), 3.16\left(1 \mathrm{H}, \mathrm{d}, \mathrm{J}=11.5 \mathrm{~Hz}, \mathrm{CH}_{2}\right), 3.30(4 \mathrm{H}, \mathrm{br}$, $\left.\mathrm{CH}_{2}\right), 3.61\left(1 \mathrm{H}, \mathrm{d}, \mathrm{J}=6.5 \mathrm{~Hz}, \mathrm{CH}_{2}\right), 3.71\left(2 \mathrm{H}, \mathrm{m}, \mathrm{CH}_{2}\right)$, $3.97(1 \mathrm{H}, \mathrm{m}, \mathrm{CH}), 4.20\left(2 \mathrm{H}, \mathrm{d}, \mathrm{J}=11.5 \mathrm{~Hz}, \mathrm{CH}_{2}\right), 4.22(1 \mathrm{H}$, br, $\left.\mathrm{CH}_{2}\right), 4.45(1 \mathrm{H}, \mathrm{m}, \mathrm{CH}), 4.80(1 \mathrm{H}, \mathrm{m}, \mathrm{CH}), 4.92(1 \mathrm{H}$, m, CH), 6.74 (2H, br, ArH), 6.86 (2H, s, ArH), $7.05(2 \mathrm{H}$, br, ArH), $7.11(2 \mathrm{H}, \mathrm{s}, \mathrm{ArH}) .{ }^{13} \mathrm{C}\left\{{ }^{1} \mathrm{H}\right\}$ NMR $\left(\mathrm{CDCl}_{3}\right): \delta 16.5$ $\left(\mathrm{CH}_{3}\right), 16.9\left(\mathrm{CH}_{3}\right), 20.7\left(\mathrm{CH}_{3}\right), 23.0(\mathrm{C}), 23.7(\mathrm{C}), 25.8$ $\left(\mathrm{CH}_{3}\right), 26.1\left(\mathrm{CH}_{3}\right), 26.3\left(\mathrm{CH}_{3}\right), 55.6\left(\mathrm{CH}_{2}\right), 58.0\left(\mathrm{CH}_{2}\right)$, 59.2 (br, $\left.\mathrm{CH}_{2}\right), 62.7$ (br, $\left.\mathrm{CH}_{2}\right), 64.1\left(\mathrm{CH}_{2}\right), 72.1(\mathrm{CH}), 73.1$ (CH), $75.7(\mathrm{CH}), 75.9(\mathrm{CH}), 122.9(\mathrm{Ar}), 123.4(\mathrm{ArH}), 124.0$ (Ar), 126.7 (br, Ar), 127.9 (ArH), 139.1 (Ar), 163.0 (ArO). Calc. (\%) for $\mathrm{C}_{35} \mathrm{H}_{56} \mathrm{~N}_{2} \mathrm{O}_{4} \mathrm{Ti}: \mathrm{C}$ 68.17, $\mathrm{H}$ 9.15, N 4.54 . Found (\%), C 68.29, H 9.28, N 4.57.

$\mathrm{Ti}(3)\left(\mathrm{O}^{\mathrm{i}} \mathrm{Pr}\right)_{2} \cdot 3 \mathrm{H}_{2}(0.54 \mathrm{~g}, 1.01 \mathrm{mmol})$ and $\mathrm{Ti}\left(\mathrm{O}^{\mathrm{i}} \mathrm{Pr}\right)_{4}$ $(0.30 \mathrm{ml}, 1.01 \mathrm{mmol})$ were dissolved in toluene $(30 \mathrm{ml})$ then heated $\left(80^{\circ} \mathrm{C}\right)$ and stirred $(16 \mathrm{~h})$. The solvent was removed in-vacuo and recrystallised from hexane to yield pale yellow crystals $(0.24 \mathrm{~g}, 0.34 \mathrm{mmol}, 34 \%) .2$ species identified in the solution state NMR spectra in an approximate 50:50 ratio, a third species is present in a negligible ratio. ${ }^{1} \mathrm{H} \mathrm{NMR}\left(\mathrm{CDCl}_{3}\right): \delta 0.39(3 \mathrm{H}, \mathrm{d}, \mathrm{J}=$ $\left.6.0 \mathrm{~Hz}, \mathrm{CH}_{3}\right), 0.55\left(3 \mathrm{H}, \mathrm{d}, \mathrm{J}=6.0 \mathrm{~Hz}, \mathrm{CH}_{3}\right), 0.72(3 \mathrm{H}, \mathrm{d}$, $\left.\mathrm{J}=6.0 \mathrm{~Hz}, \mathrm{CH}_{3}\right), 0.94\left(3 \mathrm{H}, \mathrm{d}, \mathrm{J}=6.0 \mathrm{~Hz}, \mathrm{CH}_{3}\right), 0.97(3 \mathrm{H}$, d, J = 6.0 Hz, $\left.\mathrm{CH}_{3}\right), 0.98\left(3 \mathrm{H}, \mathrm{d}, \mathrm{J}=6.0 \mathrm{~Hz}, \mathrm{CH}_{3}\right), 1.01$ $\left(6 \mathrm{H}, \mathrm{d}, \mathrm{J}=6.0 \mathrm{~Hz}, \mathrm{CH}_{3}\right), 1.26\left(18 \mathrm{H}, \mathrm{s},{ }^{\mathrm{t}} \mathrm{Bu}\right), 1.28(18 \mathrm{H}, \mathrm{s}$, $\left.{ }^{\mathrm{t}} \mathrm{Bu}\right), 1.46\left(9 \mathrm{H}, \mathrm{s},{ }^{\mathrm{t}} \mathrm{Bu}\right), 1.47\left(9 \mathrm{H}, \mathrm{s},{ }^{\mathrm{t}} \mathrm{Bu}\right), 1.48(18 \mathrm{H}, \mathrm{s}$, $\left.{ }^{\mathrm{t}} \mathrm{Bu}\right), 1.82\left(2 \mathrm{H}, \mathrm{m}, \mathrm{CH}_{2}\right), 2.23\left(3 \mathrm{H}, \mathrm{m}, \mathrm{CH}_{2}\right), 2.38(3 \mathrm{H}, \mathrm{m}$, $\left.\mathrm{CH}_{2}\right), 2.45\left(1 \mathrm{H}, \mathrm{m}, \mathrm{CH}_{2}\right), 2.72\left(2 \mathrm{H}, \mathrm{m}, \mathrm{CH}_{2}\right), 3.05(1 \mathrm{H}$, d, J = $\left.11.5 \mathrm{~Hz}, \mathrm{CH}_{2}\right), 3.23\left(2 \mathrm{H}, \mathrm{d}, \mathrm{J}=11.5 \mathrm{~Hz}, \mathrm{CH}_{2}\right), 3.44$ $\left(1 \mathrm{H}, \mathrm{d}, \mathrm{J}=14.5 \mathrm{~Hz}, \mathrm{CH}_{2}\right), 3.55\left(2 \mathrm{H}, \mathrm{m}, \mathrm{CH}_{2}\right), 3.61(2 \mathrm{H}$, $\left.\mathrm{d}, \mathrm{J}=6.5 \mathrm{~Hz}, \mathrm{CH}_{2}\right), 3.88\left(2 \mathrm{H}, \mathrm{m}, \mathrm{CH}_{2}\right), 3.97(1 \mathrm{H}, \mathrm{br}$, $\left.\mathrm{CH}_{2}\right), 4.01\left(2 \mathrm{H}, \mathrm{d}, \mathrm{J}=11.5 \mathrm{~Hz}, \mathrm{CH}_{2}\right), 4.13(1 \mathrm{H}, \mathrm{d}, \mathrm{J}=11.5$ $\left.\mathrm{Hz}, \mathrm{CH}_{2}\right), 4.17\left(1 \mathrm{H}, \mathrm{m}, \mathrm{CH}_{2}\right), 4.23(1 \mathrm{H}, \mathrm{m}, \mathrm{CH}), 4.28$ $(1 \mathrm{H}, \mathrm{m}, \mathrm{CH}), 4.51\left(1 \mathrm{H}, \mathrm{d}, \mathrm{J}=11.5 \mathrm{~Hz}, \mathrm{CH}_{2}\right), 4.55(2 \mathrm{H}$, $\mathrm{m}, \mathrm{CH}), 6.74(1 \mathrm{H}, \mathrm{d}, \mathrm{J}=2.0 \mathrm{~Hz}, \mathrm{ArH}), 6.88(2 \mathrm{H}, \mathrm{d}, \mathrm{J}=2.5$ $\mathrm{Hz}, \mathrm{ArH}), 6.90(1 \mathrm{H}, \mathrm{d}, \mathrm{J}=2.5 \mathrm{~Hz}, \mathrm{ArH}), 7.16(1 \mathrm{H}, \mathrm{d}, \mathrm{J}=$ $2.5 \mathrm{~Hz}, \mathrm{ArH}), 7.25$ (2H, d, J = 2.5 Hz, ArH), $7.27(1 \mathrm{H}, \mathrm{br}$, $\mathrm{ArH}) .{ }^{13} \mathrm{C}\left\{{ }^{1} \mathrm{H}\right\} \operatorname{NMR}\left(\mathrm{CDCl}_{3}\right): \delta 22.9\left(\mathrm{CH}_{2}\right), 23.3\left(\mathrm{CH}_{2}\right)$, $25.4\left(\mathrm{CH}_{3}\right), 25.5\left(\mathrm{CH}_{3}\right), 25.9\left(\mathrm{CH}_{3}\right), 26.1\left(\mathrm{CH}_{3}\right), 26.2$ $\left(\mathrm{CH}_{3}\right), 26.7\left(\mathrm{CH}_{3}\right), 30.0\left(\mathrm{CH}_{3}\right), 30.3\left(\mathrm{CH}_{3}\right), 30.6\left(\mathrm{CH}_{3}\right)$, $32.0\left(\mathrm{CH}_{3}\right), 34.1(\mathrm{C}), 34.2(\mathrm{C}), 35.1(\mathrm{C}), 35.3(\mathrm{C}), 35.5$ (C), $35.6(\mathrm{C}), 51.3\left(\mathrm{CH}_{2}\right), 52.6\left(\mathrm{CH}_{2}\right), 55.2\left(\mathrm{CH}_{2}\right), 55.3$ $\left(\mathrm{CH}_{2}\right), 55.6\left(\mathrm{CH}_{2}\right), 57.7\left(\mathrm{CH}_{2}\right), 58.4\left(\mathrm{CH}_{2}\right), 59.0\left(\mathrm{CH}_{2}\right)$, $63.2\left(\mathrm{CH}_{2}\right), 64.4\left(\mathrm{CH}_{2}\right), 64.6\left(\mathrm{CH}_{2}\right), 72.7(\mathrm{CH}), 72.9$ $(\mathrm{CH}), 74.9(\mathrm{CH}), 76.0(\mathrm{CH}), 121.5(\mathrm{ArH}), 122.2(\mathrm{Ar})$, 122.9 (Ar), 123.5 (Ar), 123.7 (ArH), 123.8 (ArH), 123.9 (ArH), 123.9 (ArH), 124.0 (ArH), 124.4 (ArH), 124.6 (Ar), 134.1 (Ar), 134.3 (Ar), 136.7 (Ar), 136.8 (Ar), 138.3 (Ar), 138.5 (Ar), 139.4 (Ar), 139.5 (Ar), 159.1 (ArO), 160.0 (ArO), 163.5 (ArO), 164.3 (ArO). Calc. (\%) for $\mathrm{C}_{41} \mathrm{H}_{68} \mathrm{~N}_{2} \mathrm{O}_{4} \mathrm{Ti}: \mathrm{C} 70.26, \mathrm{H}$ 9.78, N 4.00. Found (\%), C 70.19, H 9.69, N 4.12.
$\mathrm{Ti}(4)\left(\mathrm{O}^{\mathrm{i}} \mathrm{Pr}\right)_{2} .4 \mathrm{H}_{2}(0.46 \mathrm{~g}, 1.02 \mathrm{mmol})$ and $\mathrm{Ti}\left(\mathrm{O}^{\mathrm{i}} \mathrm{Pr}\right)_{4}$ $(0.30 \mathrm{ml}, 1.01 \mathrm{mmol})$ were dissolved in toluene $(30 \mathrm{ml})$ then heated $\left(80^{\circ} \mathrm{C}\right)$ and stirred $(16 \mathrm{~h})$. The solvent was removed in-vacuo and recrystallised from hexane to yield pale yellow crystals (0.16 g, $0.26 \mathrm{mmol}, 26 \%)$. 2 species identified in the solution state NMR spectra in an approximate 50:50 ratio. ${ }^{1} \mathrm{H}$ NMR $\left(\mathrm{CDCl}_{3}\right): \delta 0.41\left(3 \mathrm{H}, \mathrm{br}, \mathrm{CH}_{3}\right)$, $0.55\left(3 \mathrm{H}, \mathrm{d}, \mathrm{J}=6.0 \mathrm{~Hz}, \mathrm{CH}_{3}\right), 0.72\left(3 \mathrm{H}, \mathrm{d}, \mathrm{J}=6.0 \mathrm{~Hz}, \mathrm{CH}_{3}\right)$, $0.98\left(3 \mathrm{H}, \mathrm{d}, \mathrm{J}=6.0 \mathrm{~Hz}, \mathrm{CH}_{3}\right), 1.02\left(3 \mathrm{H}, \mathrm{d}, \mathrm{J}=6.0 \mathrm{~Hz}, \mathrm{CH}_{3}\right)$, $1.00\left(3 \mathrm{H}, \mathrm{s}, \mathrm{CH}_{3}\right), 0.98\left(6 \mathrm{H}, \mathrm{br}, \mathrm{CH}_{3}\right), 1.43\left(54 \mathrm{H}, \mathrm{s},{ }^{\mathrm{t}} \mathrm{Bu}\right)$, $1.81\left(3 \mathrm{H}, \mathrm{m}, \mathrm{CH}_{2}\right), 2.15-2.30\left(7 \mathrm{H}, \mathrm{br}, \mathrm{CH}_{2}\right), 2.22(6 \mathrm{H}, \mathrm{s}$, $\left.\mathrm{CH}_{3}\right), 2.24\left(6 \mathrm{H}, \mathrm{s}, \mathrm{CH}_{3}\right), 2.25\left(6 \mathrm{H}, \mathrm{s}, \mathrm{CH}_{3}\right), 2.30-2.45(6 \mathrm{H}$, $\left.\mathrm{m}, \mathrm{CH}_{2}\right), 2.54\left(1 \mathrm{H}, \mathrm{m}, \mathrm{CH}_{2}\right), 2.66\left(2 \mathrm{H}, \mathrm{m}, \mathrm{CH}_{2}\right), 2.78(1 \mathrm{H}$, br, $\left.\mathrm{CH}_{2}\right), 3.05\left(2 \mathrm{H}, \mathrm{d}, \mathrm{J}=12.0 \mathrm{~Hz}, \mathrm{CH}_{2}\right), 3.22(2 \mathrm{H}, \mathrm{d}, \mathrm{J}=$ $\left.11.5 \mathrm{~Hz}, \mathrm{CH}_{2}\right), 3.41\left(2 \mathrm{H}, \mathrm{t}, \mathrm{J}=13.0 \mathrm{~Hz}, \mathrm{CH}_{2}\right), 3.43(2 \mathrm{H}, \mathrm{br}$, $\left.\mathrm{CH}_{2}\right), 3.54\left(2 \mathrm{H}, \mathrm{br}, \mathrm{CH}_{2}\right), 3.68\left(2 \mathrm{H}, \mathrm{d}, \mathrm{J}=3.5 \mathrm{~Hz}, \mathrm{CH}_{2}\right)$, $3.82(2 \mathrm{H}, \mathrm{br}, \mathrm{CH}), 3.98\left(3 \mathrm{H}, \mathrm{br}, \mathrm{CH}_{2}\right), 4.05(2 \mathrm{H}, \mathrm{d}, \mathrm{J}=12.0$ $\left.\mathrm{Hz}, \mathrm{CH}_{2}\right), 4.12\left(2 \mathrm{H}, \mathrm{m}, \mathrm{CH}_{2}\right), 4.24(2 \mathrm{H}, \mathrm{m}, \mathrm{CH}), 4.51(2 \mathrm{H}$, $\left.\mathrm{d}, \mathrm{J}=14.0 \mathrm{~Hz}, \mathrm{CH}_{2}\right), 4.61(2 \mathrm{H}, \mathrm{m}, \mathrm{CH}), 5.31(1 \mathrm{H}, \mathrm{d}, \mathrm{J}=$ $\left.13.0 \mathrm{~Hz}, \mathrm{CH}_{2}\right), 6.61$ (1H, s, ArH), 6.75 (2H, s, ArH), 6.77 (2H, s, ArH ), 6.81 (1H, s, ArH), 6.97 (1H, s, ArH), 7.01 (4H, s, ArH), $7.09(1 \mathrm{H}, \mathrm{s}, \mathrm{ArH}) .{ }^{13} \mathrm{C}\left\{{ }^{1} \mathrm{H}\right\}$ NMR $\left(\mathrm{CDCl}_{3}\right): \delta$ $20.9\left(\mathrm{CH}_{3}\right), 21.0\left(\mathrm{CH}_{3}\right), 21.1\left(\mathrm{CH}_{3}\right), 22.9\left(\mathrm{CH}_{3}\right), 23.4$ $\left(\mathrm{CH}_{3}\right), 25.8\left(\mathrm{CH}_{3}\right), 25.9\left(\mathrm{CH}_{3}\right), 26.0\left(\mathrm{CH}_{3}\right), 26.3\left(\mathrm{CH}_{3}\right)$, $26.4\left(\mathrm{CH}_{3}\right), 26.9\left(\mathrm{CH}_{3}\right), 29.9\left(\mathrm{CH}_{3}\right), 30.2\left(\mathrm{CH}_{3}\right), 30.6$ $\left(\mathrm{CH}_{3}\right), 34.8(\mathrm{C}), 35.0(\mathrm{C}), 35.2(\mathrm{C}), 35.3(\mathrm{C}), 50.9\left(\mathrm{CH}_{2}\right)$, $52.6\left(\mathrm{CH}_{2}\right), 55.2\left(\mathrm{CH}_{2}\right), 55.5\left(\mathrm{CH}_{2}\right), 55.7\left(\mathrm{CH}_{2}\right), 57.8$ $\left(\mathrm{CH}_{2}\right), 58.4\left(\mathrm{CH}_{2}\right), 59.1\left(\mathrm{CH}_{2}\right), 62.9\left(\mathrm{CH}_{2}\right), 64.3\left(\mathrm{CH}_{2}\right)$, 64.4 $\left(\mathrm{CH}_{2}\right), 64.7\left(\mathrm{CH}_{2}\right), 72.7(\mathrm{CH}), 73.0(\mathrm{CH}), 75.1(\mathrm{CH})$, $75.9(\mathrm{CH}), 121.8$ (Ar), 122.9 (Ar), 124.2 (Ar), 124.6 (Ar), 124.9 (Ar), 125.3 (Ar), 125.7 (ArH), 125.9 (Ar), 126.0 (Ar), 126.9 (ArH), $127.4(\mathrm{ArH}), 127.7$ (ArH), 127.8 (ArH), 128.0 (ArH), $128.2(\mathrm{ArH}), 134.8$ (Ar), 135.0 (Ar), 137.5 (Ar), 137.6 (Ar), 134.8 (Ar), 159.1 (ArO), 159.9 (ArO), 163.5 (ArO), 164.3 (ArO). Calc. (\%) for $\mathrm{C}_{35} \mathrm{H}_{56} \mathrm{~N}_{2} \mathrm{O}_{4} \mathrm{Ti}$ : C 68.17, $\mathrm{H}$ 9.15, N 4.54. Found (\%), C 66.45, H 8.85, N 4.58.

$\mathrm{Ti}(5)\left(\mathrm{O}^{\mathrm{i}} \mathrm{Pr}\right)_{2} .5 \mathrm{H}_{2}(0.60 \mathrm{~g}, 1.01 \mathrm{mmol})$ and $\mathrm{Ti}\left(\mathrm{O}^{\mathrm{i}} \mathrm{Pr}\right)_{4}$ $(0.30 \mathrm{ml}, 1.01 \mathrm{mmol})$ were dissolved in toluene $(30 \mathrm{ml})$ then heated $\left(80^{\circ} \mathrm{C}\right)$ and stirred $(16 \mathrm{~h})$. The solvent was removed in-vacuo and recrystallised from hexane to yield pale yellow crystals (0.13 g, $0.17 \mathrm{mmol}, 17 \%) .2$ species identified in the solution state NMR spectra in an approximate 50:50 ratio. ${ }^{1} \mathrm{H} \mathrm{NMR}\left(\mathrm{CDCl}_{3}\right): \delta 0.33(3 \mathrm{H}, \mathrm{d}, \mathrm{J}=6.0$ $\left.\mathrm{Hz}, \mathrm{CH}_{3}\right), 0.55\left(3 \mathrm{H}, \mathrm{d}, \mathrm{J}=6.0 \mathrm{~Hz}, \mathrm{CH}_{3}\right), 0.67(15 \mathrm{H}, \mathrm{m}$, $\left.\mathrm{CH}_{3}\right), 0.76\left(12 \mathrm{H}, \mathrm{m}, \mathrm{CH}_{3}\right), 0.99\left(3 \mathrm{H}, \mathrm{d}, \mathrm{J}=6.0 \mathrm{~Hz}, \mathrm{CH}_{3}\right)$, $1.01\left(3 \mathrm{H}, \mathrm{d}, \mathrm{J}=6.0 \mathrm{~Hz}, \mathrm{CH}_{3}\right), 1.12\left(6 \mathrm{H}, \mathrm{d}, \mathrm{J}=6.0 \mathrm{~Hz}, \mathrm{CH}_{3}\right)$, $1.23\left(6 \mathrm{H}, \mathrm{s}, \mathrm{CH}_{3}\right), 1.26\left(12 \mathrm{H}, \mathrm{s}, \mathrm{CH}_{3}\right), 1.29\left(6 \mathrm{H}, \mathrm{s}, \mathrm{CH}_{3}\right)$, $1.39\left(3 \mathrm{H}, \mathrm{s}, \mathrm{CH}_{3}\right), 1.41\left(3 \mathrm{H}, \mathrm{s}, \mathrm{CH}_{3}\right), 1.45\left(9 \mathrm{H}, \mathrm{s}, \mathrm{CH}_{3}\right)$, $1.47\left(9 \mathrm{H}, \mathrm{s}, \mathrm{CH}_{3}\right), 1.60\left(8 \mathrm{H}, \mathrm{m}, \mathrm{CH}_{2}\right), 1.60\left(8 \mathrm{H}, \mathrm{m}, \mathrm{CH}_{2}\right)$, 1.75 - $1.95\left(6 \mathrm{H}, \mathrm{m}, \mathrm{CH}_{2}\right), 2.07\left(3 \mathrm{H}, \mathrm{m}, \mathrm{CH}_{2}\right), 2.22(4 \mathrm{H}, \mathrm{m}$, $\left.\mathrm{CH}_{2}\right), 2.30-2.50\left(4 \mathrm{H}, \mathrm{m}, \mathrm{CH}_{2}\right), 2.66\left(1 \mathrm{H}, \mathrm{m}, \mathrm{CH}_{2}\right), 2.78$ $\left(2 \mathrm{H}, \mathrm{m}, \mathrm{CH}_{2}\right), 3.06\left(1 \mathrm{H}, \mathrm{d}, \mathrm{J}=11.5 \mathrm{~Hz}, \mathrm{CH}_{2}\right), 3.20(2 \mathrm{H}, \mathrm{d}$, $\left.\mathrm{J}=11.5 \mathrm{~Hz}, \mathrm{CH}_{2}\right), 3.44\left(1 \mathrm{H}, \mathrm{d}, \mathrm{J}=14.5 \mathrm{~Hz}, \mathrm{CH}_{2}\right), 3.53(2 \mathrm{H}$, d, J = 7.5 Hz, $\left.\mathrm{CH}_{2}\right), 3.62\left(2 \mathrm{H}, \mathrm{d}, \mathrm{J}=6.5 \mathrm{~Hz}, \mathrm{CH}_{2}\right), 3.90(2 \mathrm{H}$, 
Table 4 Crystallographic parameters for $\mathrm{Ti}(2,4-6)\left(\mathrm{O}^{\mathrm{i}} \mathrm{Pr}\right)_{2}$

\begin{tabular}{|c|c|c|c|c|}
\hline Compound reference & $\mathrm{Ti}(2)\left(\mathrm{O}^{\mathrm{i}} \mathrm{Pr}\right)_{2}$ & $\mathrm{Ti}(4)\left(\mathrm{O}^{\mathrm{i}} \mathrm{Pr}\right)_{2}$ & $\mathrm{Ti}(5)\left(\mathrm{O}^{\mathrm{i}} \mathrm{Pr}\right)_{2}$ & $\mathrm{Ti}(6)\left(\mathrm{O}^{\mathrm{i}} \mathrm{Pr}\right)_{2}$ \\
\hline Chemical formula & $\mathrm{C}_{35} \mathrm{H}_{56} \mathrm{~N}_{2} \mathrm{O}_{4} \mathrm{Ti}$ & $\mathrm{C}_{35} \mathrm{H}_{56} \mathrm{~N}_{2} \mathrm{O}_{4} \mathrm{Ti}$ & $\mathrm{C}_{45} \mathrm{H}_{76} \mathrm{~N}_{2} \mathrm{O}_{4} \mathrm{Ti}$ & $\mathrm{C}_{40} \mathrm{H}_{60} \mathrm{~N}_{2} \mathrm{O}_{4} \mathrm{Ti}$ \\
\hline Formula mass & 616.72 & 616.72 & 756.95 & 680.80 \\
\hline Crystal system & Triclinic & Monoclinic & Orthorhombic & Monoclinic \\
\hline$a / \AA ̊$ & $11.2340(14)$ & $19.3720(7)$ & $10.9250(7)$ & $14.0530(2)$ \\
\hline$b / \AA ̊$ & $12.4310(17)$ & $9.6520(4)$ & $14.7200(12)$ & $13.5820(2)$ \\
\hline$c / \AA$ & $13.3470(12)$ & $20.3950(10)$ & $27.1040(14)$ & $20.1480(4)$ \\
\hline$a /^{\circ}$ & $83.037(7)$ & 90.00 & 90 & 90 \\
\hline$\beta /^{\circ}$ & $71.297(6)$ & $116.015(2)$ & 90 & $91.5280(10)$ \\
\hline$\gamma /{ }^{\circ}$ & $89.540(5)$ & 90.00 & 90 & 90 \\
\hline Unit cell volume $/ \AA^{3}$ & 1751.5(4) & $3427.0(3)$ & $4358.8(5)$ & $3844.24(11)$ \\
\hline Temperature/K & $150(2)$ & $150(2)$ & $150(2)$ & $150(2)$ \\
\hline Space group & $P \overline{1}$ & $P 2_{1} / a$ & $P 2_{1} 2_{1} 2_{1}$ & $P 2_{1} / n$ \\
\hline Z & 2 & 4 & 4 & 4 \\
\hline No. of reflections measured & 36743 & 21094 & 52730 & 66001 \\
\hline No. of independent reflections & 7965 & 5422 & 6899 & 6743 \\
\hline$R_{\text {int }}$ & 0.0877 & 0.1729 & 0.1339 & 0.1218 \\
\hline Final $R_{1}$ values $(I>2 \sigma(I))$ & 0.0564 & 0.0837 & 0.0607 & 0.0503 \\
\hline Final $w R\left(F^{2}\right)$ values $(I>2 \sigma(\Lambda))$ & 0.1265 & 0.1960 & 0.1452 & 0.1156 \\
\hline Final $R_{1}$ values (all data) & 0.1066 & 0.1272 & 0.0918 & 0.0830 \\
\hline Final $w R\left(F^{2}\right)$ values (all data) & 0.1497 & 0.2254 & 0.1651 & 0.1348 \\
\hline Goodness of fit on $F^{2}$ & 1.034 & 1.079 & 1.039 & 1.027 \\
\hline
\end{tabular}

m, $\left.\mathrm{CH}_{2}\right), 4.00\left(1 \mathrm{H}, \mathrm{br}, \mathrm{CH}_{2}\right), 4.10(3 \mathrm{H}, \mathrm{d}, \mathrm{J}=11.5 \mathrm{~Hz}$, $\left.\mathrm{CH}_{2}\right), 4.22\left(1 \mathrm{H}, \mathrm{m}, \mathrm{CH}_{2}\right), 4.25(1 \mathrm{H}, \mathrm{m}, \mathrm{CH}), 4.34(1 \mathrm{H}, \mathrm{m}$, $\mathrm{CH}), 4.54\left(1 \mathrm{H}, \mathrm{d}, \mathrm{J}=13.5 \mathrm{~Hz}, \mathrm{CH}_{2}\right), 4.59(1 \mathrm{H}, \mathrm{m}, \mathrm{CH})$, $4.68(1 \mathrm{H}, \mathrm{m}, \mathrm{CH}), 6.68(1 \mathrm{H}, \mathrm{d}, \mathrm{J}=2.0 \mathrm{~Hz}, \mathrm{ArH}), 6.83(2 \mathrm{H}$, d, J = 2.5 Hz, ArH), $6.85(1 \mathrm{H}, \mathrm{d}, \mathrm{J}=2.0 \mathrm{~Hz}, \mathrm{ArH}), 7.05$ $(1 \mathrm{H}, \mathrm{d}, \mathrm{J}=2.0 \mathrm{~Hz}, \mathrm{ArH}), 7.14(2 \mathrm{H}, \mathrm{d}, \mathrm{J}=2.5 \mathrm{~Hz}, \mathrm{ArH})$, $7.16(1 \mathrm{H}$, br, ArH $) .{ }^{13} \mathrm{C}\left\{{ }^{1} \mathrm{H}\right\}$ NMR $\left(\mathrm{CDCl}_{3}\right): \delta 9.3\left(\mathrm{CH}_{3}\right)$, 9.4 $\left(\mathrm{CH}_{3}\right), 9.7\left(\mathrm{CH}_{3}\right), 9.9\left(\mathrm{CH}_{3}\right), 22.9\left(\mathrm{CH}_{2}\right), 23.4\left(\mathrm{CH}_{2}\right)$, $25.7\left(\mathrm{CH}_{3}\right), 25.8\left(\mathrm{CH}_{3}\right), 26.2\left(\mathrm{CH}_{3}\right), 26.3\left(\mathrm{CH}_{3}\right), 26.4$ $\left(\mathrm{CH}_{3}\right), 27.0\left(\mathrm{CH}_{3}\right), 27.4\left(\mathrm{CH}_{3}\right), 27.8\left(\mathrm{CH}_{3}\right), 27.8\left(\mathrm{CH}_{3}\right)$, $28.0\left(\mathrm{CH}_{3}\right), 28.1\left(\mathrm{CH}_{3}\right), 28.2\left(\mathrm{CH}_{3}\right), 28.3\left(\mathrm{CH}_{3}\right), 28.7$ $\left(\mathrm{CH}_{3}\right), 28.7\left(\mathrm{CH}_{3}\right), 28.9\left(\mathrm{CH}_{3}\right), 29.0\left(\mathrm{CH}_{3}\right), 29.1\left(\mathrm{CH}_{3}\right)$, $29.2\left(\mathrm{CH}_{3}\right), 29.3\left(\mathrm{CH}_{3}\right), 32.9(\mathrm{C}), 33.3(\mathrm{C}), 33.8(\mathrm{C})$, $37.2\left(\mathrm{CH}_{2}\right), 37.2\left(\mathrm{CH}_{2}\right), 37.3\left(\mathrm{CH}_{2}\right), 37.4\left(\mathrm{CH}_{2}\right), 37.6$ (C), $38.3(\mathrm{C}), 38.5(\mathrm{C}), 38.9(\mathrm{C}), 38.9(\mathrm{C}), 51.1\left(\mathrm{CH}_{2}\right)$, $52.5\left(\mathrm{CH}_{2}\right), 55.4\left(\mathrm{CH}_{2}\right), 55.6\left(\mathrm{CH}_{2}\right), 57.6\left(\mathrm{CH}_{2}\right), 58.2$

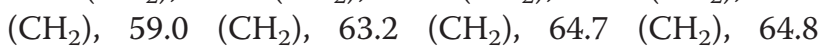
$\left(\mathrm{CH}_{2}\right), 65.2\left(\mathrm{CH}_{2}\right), 72.7(\mathrm{CH}), 73.0(\mathrm{CH}), 74.8(\mathrm{CH})$, 75.9 (CH), 120.9 (Ar), 121.9 (Ar), 123.3 (Ar), 123.4 $(\mathrm{ArH}), 123.8(\mathrm{ArH}), 124.5(\mathrm{ArH}), 124.6(\mathrm{ArH}), 125.3$ $(\mathrm{ArH}), 125.9$ (ArH), 126.5 (ArH), 132.9 (Ar), 133.0 (Ar), 134.9 (Ar), 135.1 (Ar), 136.1 (Ar), 136.2 (Ar), 137.2 (Ar), 137.4 (Ar), 158.8 (ArO), 159.7 (ArO), 163.5 (ArO), 164.1 (ArO). CHN Calc. (\%) for $\mathrm{C}_{45} \mathrm{H}_{76} \mathrm{~N}_{2} \mathrm{O}_{4} \mathrm{Ti}: \mathrm{C} 71.40, \mathrm{H}$ 10.12, $\mathrm{N}$ 3.70. Found (\%), C 71.38, H 9.97, N 3.78.
$\mathrm{Ti}(6)\left(\mathrm{O}^{\mathrm{i}} \mathrm{Pr}\right)_{2} \cdot 6^{6} \mathrm{H}_{2}(0.43 \mathrm{~g}, 1.01 \mathrm{mmol})$ and $\mathrm{Ti}\left(\mathrm{O}^{\mathrm{i}} \mathrm{Pr}\right)_{4}$ $(0.30 \mathrm{ml}, 1.01 \mathrm{mmol})$ were dissolved in toluene $(30 \mathrm{ml})$ then heated $\left(80^{\circ} \mathrm{C}\right)$ and stirred $(16 \mathrm{~h})$. The solvent was removed in-vacuo and recrystallised from hexane to yield pale yellow crystals $(0.39 \mathrm{~g}, 0.66 \mathrm{mmol}, 65 \%) .{ }^{1} \mathrm{H}$ $\operatorname{NMR}\left(\mathrm{CDCl}_{3}\right): \delta 0.63\left(6 \mathrm{H}, \mathrm{d}, \mathrm{J}=6.0 \mathrm{~Hz}, \mathrm{CH}_{3}\right), 1.61(6 \mathrm{H}$, $\left.\mathrm{d}, \mathrm{J}=6.0 \mathrm{~Hz}, \mathrm{CH}_{3}\right), 1.28\left(18 \mathrm{H}, \mathrm{s},{ }^{\mathrm{t}} \mathrm{Bu}\right), 1.72(1 \mathrm{H}, \mathrm{m}$, $\left.\mathrm{CH}_{2}\right), 1.72\left(1 \mathrm{H}, \mathrm{m}, \mathrm{CH}_{2}\right), 2.19\left(1 \mathrm{H}, \mathrm{m}, \mathrm{CH}_{2}\right), 2.28(2 \mathrm{H}$, m, $\left.\mathrm{CH}_{2}\right), 2.81\left(2 \mathrm{H}, \mathrm{d}, \mathrm{J}=6.5 \mathrm{~Hz}, \mathrm{CH}_{2}\right), 3.20(2 \mathrm{H}, \mathrm{d}, \mathrm{J}=$ $\left.11.5 \mathrm{~Hz}, \mathrm{CH}_{2}\right), 3.61\left(2 \mathrm{H}, \mathrm{d}, \mathrm{J}=6.0 \mathrm{~Hz}, \mathrm{CH}_{2}\right), 3.61(2 \mathrm{H}$, br, $\left.\mathrm{CH}_{2}\right), 3.84(1 \mathrm{H}, \mathrm{m}, \mathrm{CH}), 4.22(2 \mathrm{H}, \mathrm{d}, \mathrm{J}=11.5 \mathrm{~Hz}$, $\left.\mathrm{CH}_{2}\right), 4.82(1 \mathrm{H}, \mathrm{m}, \mathrm{CH}), 6.79(1 \mathrm{H}, \mathrm{s}, \mathrm{ArH}), 6.82(1 \mathrm{H}, \mathrm{s}$, $\mathrm{ArH}), 7.02(2 \mathrm{H}, \mathrm{d}, \mathrm{J}=2.0 \mathrm{~Hz}, \mathrm{ArH}), 7.18(1 \mathrm{H}, \mathrm{s}, \mathrm{ArH})$, $7.27(1 \mathrm{H}, \mathrm{s}, \mathrm{ArH}) .{ }^{13} \mathrm{C}\left\{{ }^{1} \mathrm{H}\right\} \mathrm{NMR}\left(\mathrm{CDCl}_{3}\right): \delta 23.0\left(\mathrm{CH}_{2}\right)$, $26.1\left(\mathrm{CH}_{3}\right), 26.2\left(\mathrm{CH}_{3}\right), 31.9\left(\mathrm{CH}_{3}\right), 34.0(\mathrm{C}), 55.3\left(\mathrm{CH}_{2}\right)$, $58.0\left(\mathrm{CH}_{2}\right), 64.1\left(\mathrm{CH}_{2}\right), 72.0(\mathrm{CH}), 73.0(\mathrm{CH}), 116.6$ (ArH), 123.6 (Ar), 125.9 (ArH), 126.5 (ArH), 139.7 (Ar), 164.1 (ArO). CHN Calc. (\%) for $\mathrm{C}_{33} \mathrm{H}_{52} \mathrm{~N}_{2} \mathrm{O}_{4} \mathrm{Ti}$ : C 67.33, H 8.90, N 4.76. Found (\%), C 67.42, H 8.89, N 4.70.

\section{Methods}

For the preparation and characterisation of metal complexes, all reactions and manipulations were performed under an inert atmosphere of argon using standard Schlenk or glovebox techniques. rac-lactide (Aldrich) was recrystallised from toluene and sublimed twice prior to 
use. All other chemicals were purchased from Aldrich. All solvents used in the preparation of metal complexes and polymerisation reactions were dry and obtained via SPS (solvent purification system). ${ }^{1} \mathrm{H}$ and ${ }^{13} \mathrm{C}\left\{{ }^{1} \mathrm{H}\right\}$ NMR spectra were recorded on a Bruker 250,300 or $400 \mathrm{MHz}$ instrument and referenced to residual solvent peaks. Coupling constants are given in Hertz. Elemental analyses were performed by Mr Stephen Boyer, London Metropolitan University. The ligands were prepared according to standard literature procedures $[3,45]$ and the purity confirmed $v i a^{1} \mathrm{H} /{ }^{13} \mathrm{C}\left\{{ }^{1} \mathrm{H}\right\}$ NMR spectroscopy and HR-MS prior to use.

\section{Polymerisation}

For solvent-free polymerisations the monomer:initiator ratio employed was $300: 1$ at a temperature of $130^{\circ} \mathrm{C}$, in all cases $1.0 \mathrm{~g}$ of $\mathrm{rac}$-lactide was used. After the reaction time methanol $(20 \mathrm{ml})$ was added to quench the reaction and the resulting solid was dissolved in dichloromethane. The solvents were removed in-vacuo and the resulting solid washed with methanol $(3 \times 50 \mathrm{ml})$ to remove any unreacted monomer. For solution polymerisations a monomer:initiator ratio of 100:1 was used. In all cases $1.0 \mathrm{~g}$ of lactide and the appropriate amount of initiator were dissolved in toluene $(10 \mathrm{ml})$ these were placed in a preheated oil bath and heated for the desired amount of time. For the melt polymerisation $1.0 \mathrm{~g}$ of lactide was used in the absence of solvent. The reaction was quenched by the addition of methanol $(20 \mathrm{ml}) .{ }^{1} \mathrm{H}$ NMR spectroscopy $\left(\mathrm{CDCl}_{3}\right)$ and GPC (THF) were used to determine tacticity and molecular weights $\left(M_{n}\right.$ and $\left.M_{\mathrm{w}}\right)$ of the polymers produced; $P_{r / m}$ (the probability of heterotactic/isotactic linkages) were determined by analysis of the methine region of the homonuclear decoupled ${ }^{1} \mathrm{H}$ NMR spectra [17]. Gel Permeation Chromatography (GPC) analyses were performed on a Polymer Laboratories PL-GPC 50 integrated system using a PLgel $5 \mu \mathrm{m}$ MIXED-D $300 \times 7.5$ $\mathrm{mm}$ column at $35^{\circ} \mathrm{C}$, THF solvent (flow rate $1.0 \mathrm{ml} / \mathrm{min}$ ). The polydispersity index (PDI) was determined from $M_{w} / M_{n}$ where $M_{\mathrm{n}}$ is the number average molecular weight and $M_{w}$ the weight average molecular weight. The polymers were referenced to polystyrene standards.

\section{Single crystal diffraction}

All data were collected on a Nonius kappa CCD diffractometer with MoKa radiation, $\lambda=0.71073 \AA$, see Table 4 . $\mathrm{T}=150(2) \mathrm{K}$ throughout and all structures were solved by direct methods and refined on $F^{2}$ data using the SHELXL-97 suite of programs [47]. The data as cif format are given in supporting information as Additional file 1 . Hydrogen atoms, were included in idealised positions and refined using the riding model. Refinements were generally straightforward with the following exceptions and points of note. $\mathrm{Ti}(\mathbf{4})\left(\mathrm{O}^{\mathrm{i}} \mathrm{Pr}\right)_{2}$ despite copious recrystallisation efforts the $R_{\text {int }}$ was higher than desirable. $\operatorname{Ti}(5)\left(\mathrm{O}^{\mathrm{i}} \mathrm{Pr}\right)_{2}$ one isopropoxide is disordered over two positions in a 60:40 ratio and despite copious recrystallisation efforts the $R_{\text {int }}$ was higher than desirable. $\mathrm{Ti}(\mathbf{6})(\mathrm{O} \mathrm{Pr})_{2}$ one isopropoxide is disordered over two positions in a 60:40 ratio, the $\mathrm{CH}_{3}$ groups of one $t \mathrm{Bu}$ are disordered over two positions in a 60:40 ratio and one toluene is disordered over two positions in a 50:50, and despite copious recrystallisation efforts the $R_{\text {int }}$ was higher than desirable.

\section{Additional file}

Additional file 1: Crystallographic data. Crystallographic data in CIF format for complexes CCDC Nos: 951134-951137.

\section{Abbreviations}

PDI: Poly dispersity index; NMR: Nuclear magnetic resonance; PLA: Polylactide; ROP: Ring opening polymerisation.

\section{Competing interests}

The authors declare that they have no competing interests.

\section{Authors' contributions}

SLH carried out the work, MDJ and SLH wrote the paper. MFM and MDJ performed the crystallographic work. All authors read and approved the final manuscript.

\section{Acknowledgments}

We wish to thank the University of Bath and the EPSRC (DTA) for funding a PhD studentship to SLH.

Received: 20 June 2013 Accepted: 29 July 2013

Published: 6 August 2013

\section{References}

1. Hancock SL, Jones MD, Langridge CJ, Mahon MF: Al(III)-homopiperazine complexes and their exploitation for the production of polyesters. New J Chem 2012, 36:1891-1896.

2. Hancock SL, Mahon MF, Jones MD: Crystallographic characterisation of $\mathrm{Ti}$ (IV) piperazine complexes and their exploitation for the ring opening polymerisation of rac-lactide. Dalton Trans 2011, 40:2033-2037.

3. Hancock SL, Mahon MF, Kociok-Kohn G, Jones MD: Homopiperazine and piperazine complexes of $\mathrm{Zr}$-IV and Hf-IV and their application to the ring-opening polymerisation of lactide. Eur J Inorg Chem 2011:4596-4602

4. Mayilmurugan R, Sankaralingam M, Suresh E, Palaniandavar M: Novel square pyramidal iron(III) complexes of linear tetradentate bis (phenolate) ligands as structural and reactive models for intradiolcleaving 3,4-PCD enzymes: Quinone formation vs. intradiol cleavage. Dalton Trans 2010, 39:9611-9625.

5. Mayilmurugan $R$, Stoeckli-Evans $H$, Suresh E, Palaniandavar $M$ Chemoselective and biomimetic hydroxylation of hydrocarbons by non-heme mu-oxo-bridged diiron(III) catalysts using m-CPBA as oxidant. Dalton Trans 2009:5101-5114.

6. Du M, Zhao XJ, Guo JH, Bu XH, Ribas J: Towards the design of linear homo-trinuclear metal complexes based on a new phenol-functionalised diazamesocyclic ligand: Structural analysis and magnetism. Eur J Inorg Chem 2005:294-304.

7. Mayilmurugan R, Harum BN, Volpe M, Sax AF, Palaniandavar M, MoeschZanetti NC: Mechanistic insight into the reactivity of oxotransferases by novel asymmetric dioxomolybdenum(VI) model complexes. Chem Eur J 2011, 17:704-713.

8. Atwood DA: Salan complexes of the group 12, 13 and 14 elements. Coord Chem Rev 1997, 165:267-296. 
9. Canali L, Sherrington DC: Utilisation of homogeneous and supported chiral metal(salen) complexes in asymmetric catalysis. Chem Soc Rev 1999, 28:85-93.

10. Cozzi PG: Metal-Salen Schiff base complexes in catalysis: practical aspects. Chem Soc Rev 2004, 33:410-421.

11. Katsuki T: Catalytic asymmetric oxidations using optically-active (Salen) manganese(III) complexes as catalysts. Coord Chem Rev 1995, 140:189-214.

12. Li WY, Zhang ZJ, Yao YM, Zhang Y, Shen Q: Control of conformations of piperazidine-bridged bis(phenolato) groups: syntheses and structures of bimetallic and monometallic lanthanide amides and their application in the polymerization of lactides. Organometallics 2012, 31:3499-3511.

13. Luo YJ, Li WY, Lin D, Yao YM, Zhang Y, Shen Q: Lanthanide alkyl complexes supported by a piperazidine-bridged Bis(phenolato) ligand: synthesis, structural characterization, and catalysis for the polymerization of L-lactide and rac-lactide. Organometallics 2010, 29:3507-3514.

14. Anderson JM, Shive MS: Biodegradation and biocompatibility of PLA and PLGA microspheres. Adv Drug Delivery Rev 2012, 64:72-82.

15. Chisholm MH, Gallucci J, Phomphrai K: Lactide polymerization by welldefined calcium coordination complexes: comparisons with related magnesium and zinc chemistry. Chem Commun 2003:48-49.

16. Chisholm MH, Gallucci JC, Phomphrai K: Well-defined calcium initiators for lactide polymerization. Inorg Chem 2004, 43:6717-6725.

17. Chamberlain BM, Cheng M, Moore DR, Ovitt TM, Lobkovsky EB, Coates GW: Polymerization of lactide with zinc and magnesium beta-diiminate complexes: Stereocontrol and mechanism. J Am Chem Soc 2001, 123:3229-3238.

18. Chisholm MH, Choojun K, Gallucci JC, Wambua PM: Chemistry of magnesium alkyls supported by 1,5,9-trimesityldipyrromethene and 2- (2,6-diisopropylphenyl)amino -4- (2,6-diisopropylphenyl)imino pent-2- ene. A comparative study. Chemical Science 2012, 3:3445-3457.

19. Chisholm MH, Gallucci J, Phomphrai K: Coordination chemistry and reactivity of monomeric alkoxides and amides of magnesium and zinc supported by the diiminato ligand $\mathrm{CH}(\mathrm{CMeNC} 6 \mathrm{H} 3-2,6-\mathrm{Pr}-\mathrm{i}(2))(2)$. A comparative study. Inorg Chem 2002, 41:2785-2794.

20. Wang Y, Zhao W, Liu DT, Li SH, Liu XL, Cui DM, Chen XS: Magnesium and zinc complexes supported by N, O-bidentate pyridyl functionalized Alkoxy Ligands: synthesis and immortal ROP of epsilon-CL and L-LA. Organometallics 2012, 31:4182-4190.

21. Jones MD, Davidson MG, Keir CG, Hughes LM, Mahon MF, Apperley DC: Zinc(II) homogeneous and heterogeneous species and their application for the ring-opening polymerisation of rac-Lactide. Eur J Inorg Chem 2009, 635:642.

22. Brignou P, Guillaume SM, Roisnel T, Bourissou D, Carpentier JF: Discrete cationic zinc and magnesium complexes for dual organic/ organometallic-catalyzed ring-opening polymerization of trimethylene carbonate. Chem Eur J 2012, 18:9360-9370.

23. Chisholm MH, Huffman JC, Phomphrai K: Monomeric metal alkoxides and trialkyl siloxides: (BDI)Mg((OBu)-Bu-t)(THF) and (BDI)Zn(OSiPh3)(THF). Comments on single site catalysts for ring-opening polymerization of lactides. J Chem Soc Dalton Trans 2001:222-224.

24. Rezayee NM, Gerling KA, Rheingold AL, Fritsch JM: Synthesis and structures of tridentate ketoiminate zinc complexes bearing trifluoromethyl substituents that act as L-lactide ring opening polymerization initiators. Dalton Trans 2013, 42:5573-5586.

25. Rieth LR, Moore DR, Lobkovsky EB, Coates GW: Single-site beta-diiminate zinc catalysts for the ring-opening polymerization of beta-butyrolactone and beta-valerolactone to poly(3-hydroxyalkanoates). J Am Chem Soc 2002, 124:15239-15248.

26. Roberts CC, Barnett BR, Green DB, Fritsch JM: Synthesis and structures of tridentate ketoiminate zinc complexes that act as L-lactide ring-opening polymerization catalysts. Organometallics 2012, 31:4133-4141.

27. Williams CK, Breyfogle LE, Choi SK, Nam W, Young VG, Hillmyer MA, Tolman WB: A highly active zinc catalyst for the controlled polymerization of lactide. J Am Chem Soc 2003, 125:11350-11359.

28. Vieira ID, Whitelaw EL, Jones MD, Herres-Pawlis S: Synergistic empirical and theoretical study on the stereoselective mechanism for the aluminum salalen complex mediated polymerization of rac-lactide. Chem Eur J 2013, 19:4712-4716.

29. Whitelaw EL, Loraine G, Mahon MF, Jones MD: Salalen aluminium complexes and their exploitation for the ring opening polymerisation of rac-lactide. Dalton Trans 2011, 40:11469-11473.
30. Bakewell C, Platel RH, Cary SK, Hubbard SM, Roaf JM, Levine AC, White AJP, Long NJ, Haaf M, Williams CK: Bis(8-quinolinolato)aluminum ethyl complexes: iso-selective initiators for rac-lactide polymerization. Organometallics 2012, 31:4729-4736.

31. Hormnirun P, Marshall EL, Gibson VC, White AJP, Williams DJ: Remarkable stereocontrol in the polymerization of racemic lactide using aluminum initiators supported by tetradentate aminophenoxide ligands. J Am Chem Soc 2004, 126:2688-2689.

32. Nomura N, Ishii R, Akakura M, Aoi K: Stereoselective ring-opening polymerization of racemic lactide using aluminum-achiral ligand complexes: Exploration of a chain-end control mechanism. J Am Chem Soc 2002, 124:5938-5939.

33. Spassky N, Wisniewski M, Pluta C, LeBorgne A: Highly stereoelective polymerization of rac- $(D, L)$-lactide with a chiral Schiff's base/aluminium alkoxide initiator. Macromol Chem Phys 1996, 197:2627-2637.

34. Yu XF, Wang ZX: Dinuclear aluminum complexes supported by amino- or imino-phenolate ligands: synthesis, structures, and ring-opening polymerization catalysis of rac-lactide. Dalton Trans 2013, 42:3860-3868.

35. Zhong ZY, Dijkstra PJ, Feijen J: (salen)Al -mediated, controlled and stereoselective ring-opening polymerization of lactide in solution and without solvent: Synthesis of highly isotactic polylactide stereocopolymers from racemic D,L-lactide. Angew Chem Int Ed Engl 2002, 41(Dijkstra, P.J):4510-4513.

36. Zhong ZY, Dijkstra PJ, Feijen J: Controlled and stereoselective polymerization of lactide: Kinetics, selectivity, and microstructures. J Am Chem Soc 2003, 125:11291-11298.

37. Kricheldorf HR: Syntheses of biodegradable and biocompatible polymers by means of bismuth catalysts. Chem Rev 2009, 109:5579-5594.

38. Whitelaw EL, Davidson MG, Jones MD: Group 4 salalen complexes for the production and degradation of polylactide. Chem Commun 2011, 47:10004-10006.

39. Whitelaw EL, Jones MD, Mahon MF: Group 4 salalen complexes and their application for the ring-opening polymerization of rac-lactide. Inorg Chem 2010, 49:7176-7181.

40. Whitelaw EL, Jones MD, Mahon MF, Kociok-Kohn G: Novel Ti(IV) and Zr(IV) complexes and their application in the ring-opening polymerisation of cyclic esters. Dalton Trans 2009:9020-9025.

41. Bonduelle C, Martin-Vaca B, Cossio FP, Bourissou D: Monomer versus alcohol activation in the 4-dimethylaminopyridine-catalyzed ringopening polymerization of lactide and lactic O-carboxylic anhydride. Chem Eur J 2008, 14:5304-5312.

42. Brown HA, De Crisci AG, Hedrick UL, Waymouth RM: Amidine-mediated zwitterionic polymerization of lactide. Acs Macro Letters 2012, 1:1113-1115.

43. Coady DJ, Fukushima K, Horn HW, Rice JE, Hedrick JL: Catalytic insights into acid/base conjugates: highly selective bifunctional catalysts for the ringopening polymerization of lactide. Chem Commun 2011, 47:3105-3107.

44. Chmura AJ, Davidson MG, Frankis CJ, Jones MD, Lunn MD: Highly active and stereoselective zirconium and hafnium alkoxide initiators for solvent-free ring-opening polymerization of rac-lactide. Chem Commun 2008:1293-1295.

45. Mohanty S, Suresh D, Balakrishna MS, Mague JT: Phosphine free diaminodiol based palladium catalysts and their application in Suzuki-Miyaura cross-coupling reactions. J Organomet Chem 2009, 694:2114-2121.

46. Gregson CKA, Blackmore IJ, Gibson VC, Long NJ, Marshall EL, White AJP: Titanium-salen complexes as initiators for the ring opening polymerisation of rac-lactide. Dalton Trans 2006:3134-3140.

47. Sheldrick GM: A short history of SHELX. Acta Crystallogr A 2008, 64:112-122.

doi:10.1186/1752-153X-7-135

Cite this article as: Hancock et al:: Monomeric Ti(IV) homopiperazine complexes and their exploitation for the ring opening polymerisation of rac-lactide. Chemistry Central Journal 2013 7:135. 